\title{
Promoção da saúde do trabalhador em pesquisas brasileiras de abordagem qualitativa: uma revisão de escopo
}

\author{
Promotion of worker's health in brazilian qualitative approach research: a scoping review
}

Promoción de la salud de los trabajadores en la investigación brasileña con un enfoque cualitativo: revisión de alcance

Recebido: 26/12/2021 | Revisado: 01/01/2022 |Aceito: 03/01/2022 | Publicado: 04/01/2022

Gerferson André Silva Costa

ORCID: https://orcid.org/0000-0003-4996-5420 Instituto René Rachou, Brasil

E-mail: gerferson.andre@gmail.com

Fabiana Goulart de Oliveira

ORCID: https://orcid.org/0000-0002-7903-1293 Universidade do Estado de Minas Gerais, Brasil

E-mail: fabiana13maio@gmail.com Celina Maria Modena

ORCID: https://orcid.org/0000-0001-5035-3427 Instituto René Rachou, Brasil

E-mail: celina.modena@fiocruz.br

\begin{abstract}
Resumo
Esta pesquisa objetivou mapear as principais evidências de pesquisas brasileiras de abordagem qualitativa, de modo a trazer as principais percepções e demandas trazidas por quem está no centro do processo produtivo: os trabalhadores. Trata-se de uma Revisão de Escopo realizada nas bases de dados, LILACS, SciElo e Biblioteca Virtual em Saúde (BVS). Dos 156 estudos encontrados, 10 compuseram esta revisão, trazendo como resultados para discussão desta pesquisa: os sentidos do trabalho e a autonomia do trabalhador, as relações interpessoais no trabalho, a questão de gênero no trabalho, território e acesso aos serviços de saúde e as condições de trabalho para promoção da saúde. Ao dialogar com as atuais políticas de promoção da saúde e saúde do trabalhador foi possível evidenciar lacunas importantes para construção de um instrumento legal que unifique, ou proponha abranger tais aspectos não contemplados, que foram discutidos nesta revisão.
\end{abstract}

Palavras-chave: Promoção da saúde; Trabalhadores; Saúde do trabalhador; Trabalho.

\begin{abstract}
This research aimed to map the main evidence of Brazilian research with a qualitative approach, in order to bring out the main perceptions and demands brought by those who are at the center of the production process: workers. This is a Scope Review carried out in the databases, LILACS, SciElo and Virtual Health Library (VHL). Of the 156 studies found, 10 composed this review, bringing as results for the discussion of this research: the meanings of work and worker autonomy, interpersonal relationships at work, the issue of gender at work, territory and access to health services and working conditions for health promotion. When dialoguing with current health promotion policies and workers' health, it was possible to highlight important gaps for the construction of a legal instrument that unifies, or proposes to cover such aspects not covered, which were discussed in this review.
\end{abstract}

Keywords: Health promotion; Workers; Occupational health; Work.

\section{Resumen}

Esta investigación tuvo como objetivo mapear las principales evidencias de la investigación brasileña con un enfoque cualitativo, con el fin de resaltar las principales percepciones y demandas planteadas por quienes están en el centro del proceso productivo: los trabajadores. Se trata de una Revisión de Alcance realizada en las bases de datos LILACS, SciElo y Biblioteca Virtual en Salud (BVS). De los 156 estudios encontrados, 10 componen esta revisión, trayendo como resultados para la discusión de esta investigación: los significados del trabajo y la autonomía del trabajador, las relaciones interpersonales en el trabajo, el tema de género en el trabajo, el territorio y el acceso a los servicios de salud y condiciones laborales para la promoción de la salud. Al dialogar con las políticas vigentes de promoción de la salud y la salud de los trabajadores, fue posible resaltar importantes brechas para la construcción de un instrumento legal que unifique o proponga cubrir los aspectos no cubiertos, los cuales fueron discutidos en esta revisión.

Palabras clave: Promoción de la salud; Trabajadores; Salud del trabajador; Trabajo. 


\section{Introdução}

A Carta de Ottawa de 1986 representa um dos principais documentos que estabelecem as bases conceituais e políticas para a promoção da saúde no mundo. Este documento destaca a multiplicidade de fatores relacionados ao processo saúdedoença e a importância da articulação dos diversos atores (Estado, comunidade, indivíduos, sistema de saúde, entre outros setores da sociedade), para o embasamento de um processo integral e equitativo (Brasil, 2002). Além disso, a promoção da saúde é definida como um processo de capacitação de indivíduos e da comunidade para melhorar e aumentar seu controle sobre a saúde, de forma participativa (Brasil, 2002). Com isso, esse texto reconhece os múltiplos determinantes de saúde (não se restringindo a cuidados médicos e paliativos) e avança na discussão sobre saúde que, anteriormente, estava limitada à relação presença/ausência de doença (Poland, 2007).

No Brasil, a definição da promoção da saúde surgiu por meio da Constituição Federal de 1988 (Brasil, 1988; Buss \& Carvalho, 2009). Ao garantir que a saúde fosse considerada um direito de todos e um dever do Estado, a promoção desse direito se daria por meio de ações e serviços acessíveis, de forma universal, e por meio de políticas sociais e econômicas (Brasil, 1988; Buss \& Carvalho, 2009). Isso significa que para promover saúde é preciso uma mudança de perspectiva, do indivíduo para a população e da visão reducionista e linear da causalidade dos fatores de risco, para uma compreensão holística, tendo em vista o contexto onde as pessoas vivem e trabalham e a relação com os fatores pessoais, ambientais e organizacionais (Dooris, 2006).

Em se tratando de promover saúde dos trabalhadores, destaca-se nesse processo, o trabalho em todos os seus aspectos: como/quando/o que/com quem/para quem se produz, a autonomia dos sujeitos envolvidos no processo produtivo, os meios disponíveis para a produção, os locais de trabalho, as relações interpessoais estabelecidas, entre outros fatores (Alcantara et al., 2020; Motalebi G. et al., 2018; Soares et al., 2021). Sabendo-se que os trabalhadores permanecem, pelo menos, um terço do seu dia em atividade laboral, este momento torna-se propício para uma política promocional de saúde, alcançando indivíduos com perfil socioeconômico semelhante (Proper \& van Oostrom, 2019; World Health Organization, 2008).

A maioria das revisões sobre este tema, trazem a promoção da saúde dos trabalhadores sumarizando e discutindo intervenções específicas sobre algum aspecto, como alimentação saudável (Lake et al., 2016; Proper \& van Oostrom, 2019), ou prática de atividade física (Cocchiara et al., 2020; Grimani et al., 2019). Mas, ainda há a necessidade de revisões mais amplas que realizem a descrição sobre o que se tem discutido sobre promoção da saúde dos trabalhadores e que possam colaborar com a construção de política na área. No Brasil, essa análise ainda não foi realizada, o que representa uma oportunidade para mapear tais estudos e trazer elementos para esta discussão.

Considerando que a compreensão dos facilitadores e barreiras presentes no processo de consolidação de uma política de promoção da saúde do trabalhador requerem uma análise em profundidade no campo dos significados e percepções, as pesquisas qualitativas podem fornecer informações valiosa (Macedo et al., 2018; Ribeiro et al., 2016). Nesse tipo de estudo, o pesquisador, em geral, assume uma posição crítica e implicada no processo, destacando a subjetividade e os sentidos reconhecidos nos sujeitos pesquisados (Creswell, 2014).

Tendo em vista a importância de pesquisas com essa abordagem e a escassez de uma discussão sobre esse tema no Brasil, esta pesquisa objetiva mapear as evidências atuais sobre a promoção da saúde do trabalhador em pesquisas qualitativas, com base no seguinte questionamento: quais aspectos da promoção da saúde do trabalhador têm sido discutidos nas pesquisas brasileiras e que podem apontar elementos importantes para criação de uma política na área?

\section{Metodologia}

Para a condução deste estudo, foi realizada uma Revisão de Escopo (Munn et al., 2018) para sumarizar os principais resultados sobre o tema. A metodologia Revisão de Escopo baseou-se nas etapas descritas por Arksey e O'Malley da 
University of York, Reino Unido (Arksey \& O’Malley, 2005). Esta metodologia consiste em cinco etapas: 1) Identificar uma questão de pesquisa; 2) Identificar estudos relevantes; 3) Selecione estudos; 4) Mapear os dados; e 5) Classificar, resumir e relatar os resultados encontrados (Arksey \& O’Malley, 2005).

Os critérios de inclusão utilizados para a seleção dos estudos foram: artigos científicos realizados no Brasil que utilizassem a abordagem qualitativa ou quanti-qualitativa nos idiomas português, inglês ou espanhol; artigos publicados no período de 2009 a 2019. As pesquisas selecionadas para análise tratavam-se de estudos originais com trabalhadores e que discutiam os resultados encontrados com implicações para a promoção da saúde. Dessa forma, estudos editoriais (cartas, comentários, notas breves), artigos de revisão, de reflexão, ou que foram realizados em outros países foram excluídos da análise.

A busca dos artigos foi realizada nas seguintes bases de dados: Scientific Electronic Library Online (SciELO); Biblioteca Virtual em Saúde (BVS), com acesso ao Base de Dados em Enfermagem (BDENF) e Index Psicologia; e Literatura Latino-Americana e do Caribe em Ciências da Saúde (LILACS). Como estratégia de localização de artigos foram combinados os seguintes Descritores em Ciências da Saúde (DeCS) com seus operadores booleanos: ("Promoção da Saúde", OR "Promoción de la Salud", OR "Health Promotion") AND (Trabalhadores, OR Grupos Profesionales, OR Occupational Groups) AND ("pesquisa qualitativa" OR "investigación cualitativa" OR "qualitative research").

Dois autores (GASC e FGO) iniciaram a busca nas bases de dados, de forma independente. Após a localização dos artigos, procedeu-se à leitura dos títulos e resumos pelos mesmos autores, excluindo as pesquisas que não atenderam aos critérios estabelecidos.

Em seguida, os artigos pré-selecionados foram lidos na íntegra e avaliada sua qualidade (Hawker et al., 2002). Nesta avaliação, foi realizada a conversão para uma pontuação numérica atribuindo as respostas de 1 ponto (ruim) a 4 pontos (ótima). Isso produziu uma pontuação para cada estudo de um mínimo de 9 pontos e um máximo de 36 pontos. Para criar as notas gerais de qualidade, usamos as seguintes definições: alta qualidade, 30-36 pontos; média qualidade, 24-29 pontos; baixa qualidade, 9-24 pontos. Estudos classificados como baixa qualidade foram excluídos da amostra final, pois buscou-se trazer uma síntese com estudos que apresentaram o maior rigor na descrição de como a pesquisa foi conduzida.

Finalizada essa avaliação mais ampla e crítica, foi obtida então a amostra final. Na Figura 1, descrevemos o processo de seleção de artigos de forma objetiva nos moldes do fluxograma PRISMA (Tricco et al., 2018) e na Figura 2 a avaliação de qualidade dos artigos. 
Figura 1: Seleção de artigos para revisão (fluxograma PRISMA).

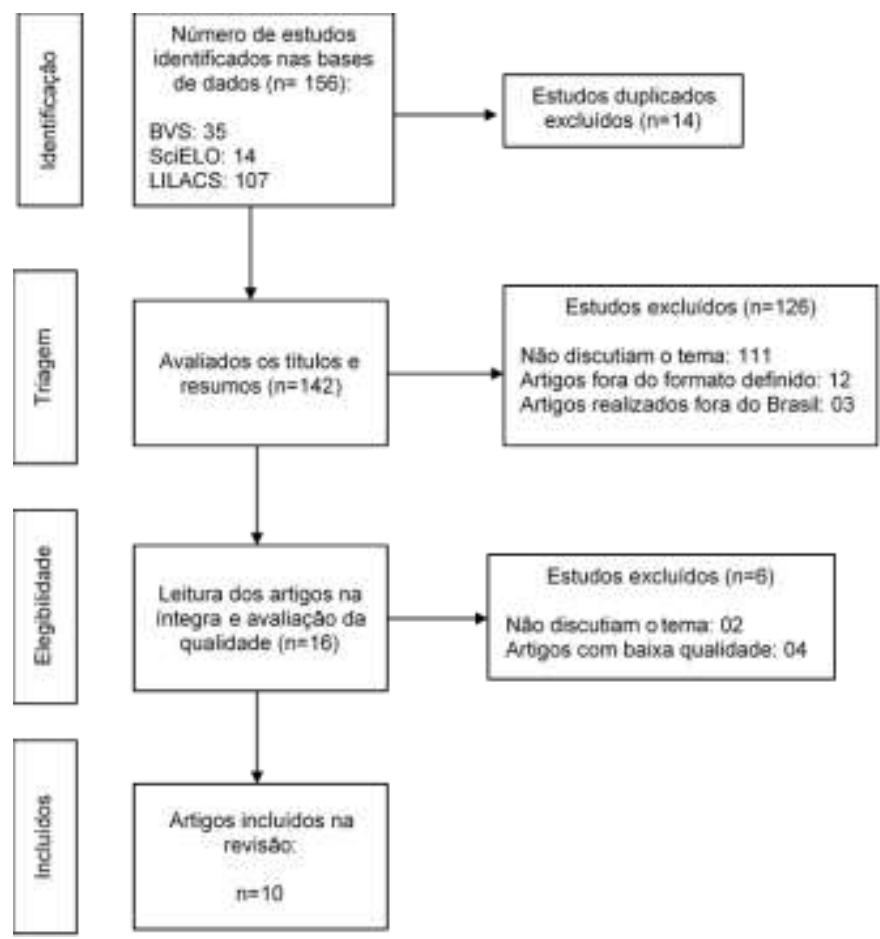

Fonte: Autores.

Quadro 1: Avaliação da qualidade dos artigos.

\begin{tabular}{|c|c|c|}
\hline Artigo/Autores & Total de pontos & Avaliação final \\
\hline (Michelin et al., 2016) & 28 & Média qualidade \\
\hline (Ferreira et al., 2015) & 26 & Média qualidade \\
\hline (Feliciano et al., 2011) & 30 & Alta qualidade \\
\hline (Gomes et al., 2015) & 26 & Média qualidade \\
\hline (Leônidas \& Melo, 2018) & 25 & Média qualidade \\
\hline (Coelho et al., 2016) & 27 & Média qualidade \\
\hline (Jaskowiak \& Fontana, 2015) & 28 & Média qualidade \\
\hline (Bezerra et al., 2014) & 26 & Alta qualidade \\
\hline (Santos \& Hennington, 2013) & 32 & Média qualidade \\
\hline (Silva et al., 2015) & 29 & \\
\hline
\end{tabular}

Fonte: Autores.

Os 10 estudos que compuseram esta análise final, partiu da concordância de pelo menos dois dos autores (GASC, FGO e CMM) desta revisão. Os artigos foram lidos exaustivamente pelos autores, buscando avaliar os principais resultados para uma avaliação crítica.

\section{Resultados}

A pesquisa nas bases de dados identificou 156 possíveis artigos para análise. Deste número 14 eram artigos duplicados. Dos 142 artigos que restaram, após a leitura dos títulos e resumos, foram excluídos mais 126 artigos (88,7\%), dos quais: $88,1 \%$ não discutiam o tema, $9,5 \%$ estavam fora do formato (pesquisa documental, dissertações/teses, revisões, etc.), $2,4 \%$ dos estudos foram realizados fora do Brasil. A etapa final consistiu na leitura na íntegra dos artigos restantes ( $\mathrm{n}=16) \mathrm{e}$ 
avaliação da qualidade (Hawker et al., 2002). Desta amostra, foram excluídos dois artigos (12,5\%) por não discutirem o tema e quatro artigos $(25 \%)$ por apresentarem uma pontuação na avaliação de qualidade inferior a 24 pontos, permanecendo nesta revisão um total de 10 artigos.

\subsection{Perfil dos trabalhadores participantes}

A maioria dos estudos (40\%) tiveram como participantes os profissionais da saúde: toda a equipe de um centro de saúde (Michelin et al., 2016), equipe de enfermagem de uma Unidade de Terapia Intensiva (Ferreira et al., 2015), médicos da Estratégia de Saúde da Família (Feliciano et al., 2011) e os Agentes Comunitários de Saúde (Gomes et al., 2015). E os outros estudos pesquisaram trabalhadores diversos, como: usuários do Centro de Referência em Saúde do Trabalhador (CEREST) e representantes de sindicato (Leônidas \& Melo, 2018), catadoras de materiais recicláveis (Coelho et al., 2016), agentes penitenciários (Jaskowiak \& Fontana, 2015), trabalhadores de uma comunidade portuária (Bezerra et al., 2014), Trabalhadores Rurais Sem Terra (Santos \& Hennington, 2013) e feirantes (Silva et al., 2015).

Dos 10 estudos selecionados, seis tiveram, majoritariamente, mulheres entrevistadas (Coelho et al., 2016; Feliciano et al., 2011; Ferreira et al., 2015; Gomes et al., 2015; Michelin et al., 2016; Silva et al., 2015). Destes 6 estudos, quatro entrevistaram profissionais da saúde (Feliciano et al., 2011; Ferreira et al., 2015; Gomes et al., 2015; Michelin et al., 2016). Apenas um estudo ressaltou a diferença na proporção entre entrevistados homens/mulheres, dada a natureza do trabalho no campo (Santos \& Hennington, 2013). E um estudo não descreveu tal proporção em relação ao sexo (Bezerra et al., 2014). No que diz respeito a faixa etária dos entrevistados, ela variou de 18 a 75 anos, e apenas um estudo não fez tal descrição (Santos \& Hennington, 2013).

\subsection{Principais resultados dos estudos selecionados}

O Quadro 2 traz os estudos selecionados com os respectivos objetivos de cada pesquisa, a descrição dos trabalhadores participantes e os principais resultados relatados pelos autores.

Quadro 2: Estudos selecionados para a revisão e os principais resultados

\begin{tabular}{|c|c|c|c|c|}
\hline Referência & Ano & Objetivo & $\begin{array}{l}\text { Trabalhadores } \\
\text { participantes }\end{array}$ & Principais Resultados \\
\hline $\begin{array}{l}\text { (Michelin et } \\
\text { al., 2016) }\end{array}$ & 2016 & $\begin{array}{l}\text { Compreender os limites do } \\
\text { quotidiano apontados pelos } \\
\text { trabalhadores da Atenção } \\
\text { Básica para a promoção de } \\
\text { sua saúde. }\end{array}$ & $\begin{array}{l}\mathrm{N}=18 \text { participantes } \\
\text { (trabalhadores de um } \\
\text { centro de saúde do } \\
\text { muncípio de } \\
\text { Florianópolis); F: } 16, \\
\text { M: } 2\end{array}$ & $\begin{array}{l}\text {-Os limites que dificultam a promoção da saúde foram } \\
\text { definidos em duas vertentes: a falta e oxcesso; } \\
\text {-Falta tempo e/ou falta saber administrar o tempo para o } \\
\text { lazer e estudar; } \\
\text {-Falta companheirismo na equipe de trabalho; } \\
\text {-Falta fazer exercício físico; } \\
\text {-O excesso de tarefas diárias e carga horária no trabalho; } \\
\text {-Outros excessos: a gula, a preguiça e o } \\
\text { trânsito/congestionamento. }\end{array}$ \\
\hline $\begin{array}{l}\text { (Ferreira et } \\
\text { al., 2015) }\end{array}$ & 2015 & $\begin{array}{l}\text { Identificar a percepção dos } \\
\text { trabalhadores de enfermagem } \\
\text { sobre o cuidado de si; } \\
\text { descrever as ações que os } \\
\text { trabalhadores realizam para } \\
\text { cuidarem de si; e discutir as } \\
\text { repercussões do cuidado de } \\
\text { si para a saúde destes } \\
\text { trabalhadores. }\end{array}$ & $\begin{array}{l}\mathrm{N}=10 \text { ( } 3 \text { enfermeiros, } \\
2 \text { residentes de } \\
\text { enfermagem e } 5 \\
\text { técnicos de } \\
\text { enfermagem }) ; \mathrm{F}: 9, \\
\mathrm{M}: 1\end{array}$ & $\begin{array}{l}\text {-Os entrevistados relataram a necessidade de tornar o } \\
\text { ambiente de trabalho acolhedor e do acompanhamento da } \\
\text { saúde individual dos trabalhadores, por meio de exames; } \\
\text {-Na perspectiva do cuidado de si para a promoção da saúde, } \\
\text { os trabalhadores relatam a necessidade de realização de } \\
\text { atividade física e de uma alimentação saudável. O lazer (ir } \\
\text { ao cinema, show, teatro, etc.) também é destacado como um } \\
\text { fator importante para o cuidado de si; } \\
\text {-Quando esse cuidado de si não ocorre, há a possibilidade de } \\
\text { prejudicar a qualidade da assistência de enfermagem. }\end{array}$ \\
\hline
\end{tabular}




\begin{tabular}{|c|c|c|c|c|}
\hline $\begin{array}{c}\text { (Feliciano et } \\
\text { al., 2011) }\end{array}$ & 2011 & $\begin{array}{l}\text { Compreender como os } \\
\text { conflitos com a instituição e } \\
\text { desacordos quanto às } \\
\text { atribuições de membros da } \\
\text { equipe, são apreendidos e } \\
\text { reconstruídos pelos médicos } \\
\text { da Saúde da Família, sob a } \\
\text { perspectiva do "burnout". }\end{array}$ & $\begin{array}{l}\mathrm{N}=24 \text { médicos, }(12 \\
\text { pediatras, } 8 \text { clínicos, } \\
01 \text { psiquiatra, } 01 \\
\text { infectologista, } 01 \\
\text { tocoginecologista e } 01 \\
\text { anestesiologista); F: } \\
\text { 16, M: } 8\end{array}$ & $\begin{array}{l}\text {-Os resultados apontam para um comprometimento da saúde } \\
\text { mental dos médicos entrevistados. Tal comprometimento } \\
\text { está relacionado a discrepância do que idealizaram fazer na } \\
\text { Estratégia de Saúde da Família (ESF) e o que são cobrados } \\
\text { para fazer; } \\
\text {-Soma-se a isso a falta de capacitação para lidar com todos } \\
\text { os programas da ESF, conflitos com a equipe (devido a falta } \\
\text { de confiança na competência dos colegas); } \\
\text {-Como estratégia de enfrentamento dessas adversidades, os } \\
\text { médicos ou pensam em desistir do emprego, ou afastam de } \\
\text { situações que relatam não ter domínio para lidar (por } \\
\text { exemplo: recusam-se a atender gestantes na ESF). }\end{array}$ \\
\hline $\begin{array}{c}\text { (Gomes et } \\
\text { al., 2015) }\end{array}$ & 2015 & $\begin{array}{l}\text { Descrever a percepção dos } \\
\text { agentes comunitários de } \\
\text { saúde sobre riscos e agravos } \\
\text { relacionados às suas } \\
\text { atividades ocupacionais; }\end{array}$ & $\mathrm{N}=7$ ACS; F: 6, M: 1 & $\begin{array}{l}\text {-Os ACS relataram péssimas condições de sanitárias em } \\
\text { certas áreas de trabalho (com esgoto a céu aberto); } \\
\text {-Relataram também o medo da violência da região onde } \\
\text { trabalham; } \\
\text {-Relatam medo de se infectarem com doenças (hanseníase e } \\
\text { tuberculose); } \\
\text {-Apontaram a falta de instrumentos para o trabalho } \\
\text { (equipamentos de proteção individual, crachá e farda); } \\
\text {-E afirmam que estão sobrecarregados, já que atendem um } \\
\text { número de famílias superior ao recomendado e são } \\
\text { procurados até nos finais de semana pelos moradores. }\end{array}$ \\
\hline $\begin{array}{l}\text { (Leônidas \& } \\
\text { Melo, 2018) }\end{array}$ & 2018 & $\begin{array}{l}\text { Avaliar o Centro de } \\
\text { Referência em Saúde do } \\
\text { Trabalhador estadual do } \\
\text { Ceará (CEREST-CE) a partir } \\
\text { das crenças dos } \\
\text { representantes dos sindicatos } \\
\text { e usuários. }\end{array}$ & $\begin{array}{c}\mathrm{N}=8 \text { (3 representantes } \\
\text { dos sindicatos e } 5 \\
\text { usuários do serviço); } \\
\mathrm{M}: 5, \mathrm{~F}: 3\end{array}$ & $\begin{array}{l}\text {-Para os entrevistados, o CEREST contribui com a } \\
\text { identificação do nexo causal (adoecimento x trabalho), com } \\
\text { a orientação dos trabalhadores sobre seus direitos, formação } \\
\text { de trabalhadores do SUS e com visitas às empresas sob } \\
\text { solicitação dos sindicatos; } \\
\text {-Entretanto, os usuários e representantes dos sindicatos } \\
\text { dizem não saber qual é o papel do CEREST, destacando a } \\
\text { falta de divulgação do Centro para a Sociedade; } \\
\text {-Os usuários dizem que conheceram o CEREST por } \\
\text { indicação de colegas e encaminhamento do sindicato de } \\
\text { trabalhadores adoecidos. } \\
\text {-Os entrevistados apontam não haver integração entre o } \\
\text { CEREST e outras instâncias do Sistema Único de Saúde. }\end{array}$ \\
\hline $\begin{array}{l}\text { (Coelho et } \\
\text { al., 2016) }\end{array}$ & 2016 & $\begin{array}{l}\text { Compreender o risco de } \\
\text { adoecimento relacionado ao } \\
\text { trabalho e as estratégias } \\
\text { defensivas em mulheres } \\
\text { catadoras de materiais } \\
\text { recicláveis. }\end{array}$ & $\begin{array}{l}\mathrm{N}=7 \text { mulheres } \\
\text { catadoras de materiais } \\
\text { recicláveis }\end{array}$ & $\begin{array}{l}\text {-As catadoras relatam estresse no trabalho, dada as } \\
\text { características da tarefa que requerem muito esforço físico } \\
\text { (ficar muito tempo em pé, locomover-se constantemente, } \\
\text { pouco tempo de pausa, carregar peso); } \\
\text {-Tal estresse afeta o convívio familiar; } \\
\text {-Como mecanismos de defesa, buscam separar questões do } \\
\text { trabalho e questões de casa, ou negam/banalizam o } \\
\text { adoecimento; } \\
\text {-Aparece também como estratégia de defesa a rede de } \\
\text { confiança estabelecida entre as colegas (relatam que se } \\
\text { sentem mais à vontade de falar certas coisas com as colegas } \\
\text { do que com os maridos). }\end{array}$ \\
\hline $\begin{array}{l}\text { (Jaskowiak } \\
\text { \& Fontana, } \\
\text { 2015) }\end{array}$ & 2015 & $\begin{array}{l}\text { Conhecer as condições de } \\
\text { trabalho de agentes } \\
\text { penitenciários e os reflexos } \\
\text { do exercício da atividade } \\
\text { laboral em sua saúde. }\end{array}$ & $\begin{array}{c}\mathrm{N}=26 \text { agentes } \\
\text { penitenciários; } \mathrm{M}: 17 \text {, } \\
\mathrm{F}: 9\end{array}$ & $\begin{array}{l}\text {-Os entrevistados relataram as condições precárias de } \\
\text { trabalho, como ausência de EPI, compra de material de } \\
\text { trabalho com recurso próprio e o medo da morte, de doenças } \\
\text { infectocontagiosas e da violência dentro e fora do cárcere; } \\
\text {-Apesar de reconhecerem o seu papel na ressocialização do } \\
\text { preso, sentem-se tristes com a reincidência e a insuficiência } \\
\text { do Estado para lidar com a violência; } \\
\text {-Relataram também a importância da união entre os colegas } \\
\text { para enfrentar os momentos difíceis no trabalho; } \\
\text {-Apesar de terem buscado este emprego por conta da } \\
\text { estabilidade financeira, relataram a falta de perspectiva para } \\
\text { progressão na carreira e o adoecimento mental (depressão) } \\
\text { também como problemas. }\end{array}$ \\
\hline
\end{tabular}




\begin{tabular}{|c|c|c|c|c|}
\hline $\begin{array}{c}\text { (Bezerra et } \\
\text { al., 2014) }\end{array}$ & 2014 & $\begin{array}{l}\text { Este estudo discute aspectos } \\
\text { do desenvolvimento } \\
\text { econômico e as implicações } \\
\text { no trabalho, ambiente e } \\
\text { saúde em comunidades } \\
\text { circunvizinhas ao Complexo } \\
\text { Industrial e Portuário do } \\
\text { Pecém no Ceará. }\end{array}$ & $\begin{array}{l}\text { 01 Grupo focal com } \\
\text { N=13 participantes } \\
\text { (agricultor, duas } \\
\text { donas de casa, duas } \\
\text { profissionais da } \\
\text { saúde, uma } \\
\text { professora, três } \\
\text { estudantes, duas } \\
\text { lideranças } \\
\text { comunitárias, uma } \\
\text { artesã e um } \\
\text { trabalhador das } \\
\text { empresas); não houve } \\
\text { descrição clara do } \\
\text { gênero dos } \\
\text { participantes }\end{array}$ & $\begin{array}{l}\text {-Os entrevistados relatam a incerteza de permanência no } \\
\text { local onde vivem, como fator de angústia e adoecimento; } \\
\text {-Os entrevistados possuem um vínculo com o território, um } \\
\text { projeto de vida e de sustento para os filhos; } \\
\text {-A inserção de atividades fora da cultura local ameaça a } \\
\text { identidade coletiva - o emprego dos carnaubais; } \\
\text {-As modificações no território afetam o trabalho de homens } \\
\text { e mulheres: os homens deixam de trabalhar nos carnaubais e } \\
\text { sem estes, as mulheres deixam de trabalhar nas farinhadas e } \\
\text { isso afeta a cultura local que é motivo de orgulho para a } \\
\text { comunidade; } \\
\text {-Destacam também a falta de previsão da permanência nos } \\
\text { empregos, visto que a mão de obra dos moradores não está } \\
\text { sendo capacitada para as etapas mais especializadas das } \\
\text { empresas. }\end{array}$ \\
\hline $\begin{array}{l}\text { (Santos \& } \\
\text { Hennington, } \\
\text { 2013) }\end{array}$ & 2013 & $\begin{array}{l}\text { Analisar os modos de vida e } \\
\text { significados atribuídos por } \\
\text { assentados do Movimento } \\
\text { dos Trabalhadores Rurais } \\
\text { Sem Terra (MST) à saúde e } \\
\text { suas relações com o trabalho, } \\
\text { e identificar estratégias } \\
\text { desenvolvidas pelos } \\
\text { trabalhadores para manter } \\
\text { e/ou promover a saúde. }\end{array}$ & $\begin{array}{l}\mathrm{N}=11 \text { nas entrevistas } \\
\text { semiestruturadas (M: } \\
8 \text { e F: } 3 \text { ). E N=8 no } \\
\text { grupo focal (M: } 6 \text { e F: } \\
\text { 2) }\end{array}$ & $\begin{array}{l}\text {-O trabalho encontra sentidos diferentes quando se trata de } \\
\text { "trabalhar para si" e "trabalhar para o outro"; "trabalhar para } \\
\text { si", ou seja, no assentamento onde vivem representa ter } \\
\text { autonomia do que e como fazer (apesar de reconhecerem } \\
\text { que o mercado impõe regras de padronização de produção } \\
\text { dos alimentos - como uso de agrotóxicos); "trabalhar para o } \\
\text { outro", ou seja, trabalhar para outras pessoas é visto como } \\
\text { alternativa para suprir necessidades de sobrevivência e } \\
\text { consumo dos camponeses; } \\
\text {-Em ambas as situações há pontos negativos: no trabalho pra } \\
\text { si é relatada a questão do uso de agrotóxicos e exposição às } \\
\text { intempéries climáticas; e no trabalho para o outro além } \\
\text { dessas questões, soma-se os problemas do ritmo de trabalho } \\
\text { intenso e a postura inadequada que culminam com mais } \\
\text { desgaste físico. } \\
\text {-Os efeitos negativos à saúde são gerenciados controlando } \\
\text { alguns aspectos do trabalho (fracionam as doses de } \\
\text { agrotóxicos para reduzir a exposição e evitam reduzem o } \\
\text { trabalho em dias de adversidade climáticas - calor intenso e } \\
\text { ventania) e buscando atendimento no SUS e de forma } \\
\text { complementar com a fitoterapia, devido às dificuldades de } \\
\text { acesso aos serviços de saúde. }\end{array}$ \\
\hline $\begin{array}{l}\text { (Silva et al., } \\
\text { 2015) }\end{array}$ & 2015 & $\begin{array}{l}\text { Conhecer as percepções dos } \\
\text { feirantes com hipertensão } \\
\text { arterial sobre o adoecimento } \\
\text { crônico. }\end{array}$ & $\begin{array}{c}\mathrm{N}=10 \text { feirantes }(\mathrm{F}: 9 \mathrm{e} \\
\mathrm{M}: 1)\end{array}$ & $\begin{array}{l}\text {-Os feirantes relatam a percepção de que o estresse do dia a } \\
\text { dia afeta a sua pressão arterial; } \\
\text {-Há um desconhecimento da etiologia e tratamento da } \\
\text { doença (tomam anti-hipertensivos somente quando sentem } \\
\text { algum sintoma); } \\
\text {-Os feirantes relatam sofrimento ao se privarem de comer } \\
\text { alimentos que reconhecem prejudicar a sua pressão arterial; } \\
\text {-As condições de trabalho são apontadas como prejudiciais à } \\
\text { pressão arterial: ficam muito tempo em pé e só há alimentos } \\
\text { salgados e "com gordura" e sua jornada de trabalho varia de } \\
5 \text { a } 12 \text { horas diárias. }\end{array}$ \\
\hline
\end{tabular}

Legenda: N= número total de participantes; F = Feminino; M= Masculino. Fonte: Autores.

\section{Discussão}

Os dados descritos nessa revisão evidenciam que diversos aspectos tem sido discutidos em pesquisas com a temática da promoção da saúde do trabalhador, no Brasil. Os temas discutidos nos artigos selecionados trouxeram elementos importantes para discussão de uma futura política na área. Foram eles: os sentidos do trabalho (Feliciano et al., 2011; Jaskowiak \& Fontana, 2015) e a autonomia do trabalhador (Santos \& Hennington, 2013); o território (Bezerra et al., 2014) e o acesso aos serviços de saúde (Leônidas \& Melo, 2018; Santos \& Hennington, 2013); as relações interpessoais no trabalho (Coelho et al., 2016; Feliciano et al., 2011; Ferreira et al., 2015; Jaskowiak \& Fontana, 2015; Michelin et al., 2016); a questão de gênero no trabalho (Coelho et al., 2016; Santos \& Hennington, 2013); e as condições de trabalho para a promoção da saúde do trabalhador (Coelho et al., 2016; Gomes et al., 2015; Michelin et al., 2016; Silva et al., 2015). 


\subsection{Os sentidos do trabalho e a autonomia do trabalhador}

Pensar em promover saúde do trabalhador requer discutir os sentidos do trabalho. O trabalho pode ser um fator de adoecimento, ao impor uma organização do trabalho com ritmo elevado de tarefas e que não proporciona oportunidades de aprendizado ao trabalhador (Ziliotto \& Jaques, 2017). Mas também, o trabalho pode representar um elemento promotor de saúde, principalmente, quando há possibilidades de adquirir novas experiências, de transformar o trabalho e, consequentemente, de se transformar nesse processo (Viana \& Machado, 2011). Um trabalho com sentido pode ser definido como aquele que é organizado e que contribui de alguma forma com a instituição empregadora e/ou sociedade. Para ter sentido o trabalho também precisa proporcionar prazer na realização das tarefas, ser capaz de resolver problemas e com autonomia dos trabalhadores (Morin, 2002).

Sendo a autonomia do trabalhador um aspecto descrito na sua relação com a saúde, também é importante destaca-la nessa discussão (Cardoso \& Morgado, 2019; Lacaz, 2000; Santos et al., 2021). É da relação da norma com o que é possível de se fazer, no e para o trabalho, que são oportunizados os aprendizados, as recriações do modo de fazer, as produções de sentido do trabalho, e consequentemente, a produção de saúde. É preciso valorizar a experiência do trabalhador e discutir as formas de alcançar os objetivos da produção, de modo a não comprometer a sua saúde neste processo (Cardoso \& Morgado, 2019; Lacaz, 2000).

Na pesquisa realizada com os assentados do Movimento dos Trabalhadores Rurais Sem Terra (Santos \& Hennington, 2013) a questão da autonomia (trabalhar para si) ficou evidente. Ao mesmo tempo em que os trabalhadores eram forçados pelo mercado a utilizar agrotóxicos nas plantações, eles buscavam minimizar o efeito de sua exposição a esses agentes avaliando as condições climáticas do dia, bem como fracionavam as doses ao longo da semana. Mesmo que limitadas, foram estratégias proporcionadas pela autonomia de trabalhar em seu território, característica inexistente, quando trabalhavam para terceiros.

\subsection{Território e acesso aos serviços de saúde}

O território ganha uma relevância complexa, principalmente no campo da saúde pública. Apesar do seu sentido ser destacado muitas vezes como suporte à organização de práticas e serviços de saúde (Amaral et al., 2021; Silva et al., 2021), na perspectiva de promoção da saúde do trabalhador ressalta-se a necessidade de colocá-lo no patamar dos usos desses território (Santos, 2005) para produção de vida e saúde, como bem destacado nos artigos com os Trabalhadores Sem Terra (Santos \& Hennington, 2013) e a Comunidade Portuária (Bezerra et al., 2014). O trabalho desenvolvido no território tem um valor cultural e simbólico importante e que deve ser investigado, antes de propor intervenções desvinculadas da realidade local.

Em se tratando dos serviços de saúde do trabalhador, nos mais diversos níveis de atenção, esta revisão trouxe para discussão a questão do acesso. Sabendo-se das atividades produtivas que ocorrem no território, os serviços de saúde da Atenção Primária em Saúde, ali localizados, devem funcionar como uma porta de entrada do trabalhador para as ações em promoção da saúde. A avaliação do CEREST trazida nesta revisão (Leônidas \& Melo, 2018) trouxe a dificuldade de divulgação desta unidade especializada em saúde do trabalhador para a sociedade, a dificuldade de articulação com outras unidades de atenção à saúde no Sistema Único de Saúde (referência e contrarreferência) e a restrição de horário para atendimento (somente diurno). Promover saúde do trabalhador requer pensar nos desafios atuais de articulação intra/intersetorial bem como de capacitação dos trabalhadores que atuam nessas instâncias, numa perspectiva integral do cuidado (Camara et al., 2020), principalmente daqueles que possuem um acesso dificultado pela localização de sua residência/trabalho, como é o caso dos Trabalhadores Sem Terra também descritos nesta revisão. 


\subsection{As relações interpessoais no trabalho}

Outro aspecto destacado nesta revisão foi a discussão das relações e dinâmicas interpessoais para execução do trabalho. Como descrito em um dos artigos desta revisão (Feliciano et al., 2011), a falta de confiança na equipe e não ter suas percepções valorizadas no trabalho podem provocar o adoecimento dos trabalhadores. Em contrapartida, outros artigos sinalizaram efeitos positivos da rede de apoio formada pelos trabalhadores no enfrentamento das situações de trabalho, bem como de suporte para questões pessoais (Coelho et al., 2016; Jaskowiak \& Fontana, 2015). Dessa forma, promover o respeito, a valorização e o diálogo no trabalho podem trazer consequências positivas para a saúde dos trabalhadores (Pennisi et al., 2020).

\subsection{A questão de gênero no trabalho}

Apesar de nenhum artigo ter trazido de forma explícita a discussão sobre questões de gênero, trabalho e saúde, não se pode negar as diferenças encontradas nos artigos incluídos nesta revisão. Dos 10 artigos incluídos, 6 trouxeram majoritariamente mulheres entrevistadas (Coelho et al., 2016; Feliciano et al., 2011; Ferreira et al., 2015; Gomes et al., 2015; Michelin et al., 2016; Silva et al., 2015), sendo um deles realizado, exclusivamente, com mulheres (Coelho et al., 2016). Entretanto, tal informação está longe de representar uma maior visibilidade das mulheres no trabalho, dada a natureza dos dados aqui discutida.

A importância de destacar a questão de gênero na revisão é, justamente, pelo seu impacto na saúde e nas relações produtivas, de uma forma geral: mulheres possuem uma carga de trabalho maior, quando associado ao trabalho doméstico e, por isso, possuem uma maior chance de adoecimento (Araújo et al., 2006; Hirata \& Kergoat, 2008); e uma baixa representatividade de mulheres no trabalho tem sido apontada como uma menor chance para inovação para o trabalho, dada a pouca diversidade de indivíduos no local de trabalho (Patel \& Moonesinghe, 2020). Apesar de trazer o gênero, basicamente, na perspectiva homens versus mulheres, ressalta-se que ele pode ser definido, de uma forma mais ampla, como um elemento das relações sociais representada nas diferenças observáveis entre os sexos e como uma forma de representar as relações de poder (Scott, 1990). Para avançar nas questões de promoção da saúde do trabalhador e da trabalhadora, a discussão sobre gênero deve ganhar destaque, pois ela trará informações importantes sobre a aquisição de novas experiências para o trabalho e sobre a valorização/desvalorização social de determinados grupos (Araújo et al., 2019).

Apenas um artigo desta revisão justificou a diferença de entrevistados, em relação ao gênero (Santos \& Hennington, 2013), dada a subalternidade das mulheres no campo. Como não foi foco de o artigo discutir tal questão, a pesquisa pode ter invisibilizado questões importantes na perspectiva gênero dos trabalhadores sem terra.

\subsection{Condições de trabalho para a promoção da saúde}

Foram relatadas também nesta revisão aspectos das condições de trabalho e sua relação com a saúde do trabalhador. A Organização Internacional do Trabalho define condições de trabalho de forma abrangente, destacando a questão do tempo de trabalho (jornada de trabalho, turno e período de descanso), a remuneração e as condições físicas do local de trabalho, bem como as exigências de ordem mental para o trabalho (Organização Internacional do Trabalho, 2018). Uma longa jornada de trabalho (Silva et al., 2015), ou com muito esforço físico (Coelho et al., 2016; Gomes et al., 2015) foram relatados nas falas dos trabalhadores como um aspecto negativo, relacionado à dor (Coelho et al., 2016; Gomes et al., 2015), ou outras alterações fisiológicas (Silva et al., 2015).

Nessa perspectiva ampla de condições de trabalho e sabendo que a maioria dos trabalhadores passam boa parte do dia fora do domicílio, a disponibilidade de alimentos saudáveis também integra uma proposição básica para a promoção da saúde. No estudo com os feirantes (Silva et al., 2015), uma das suas falas destacavam a falta de alimentos saudáveis, tendo em vista a sua condição crônica de saúde: a hipertensão arterial. Um estudo realizado em condados noruegueses para verificar fatores 
associados ao consumo de vegetais no local de trabalho identificou que a cultura organizacional e a disponibilidade de tais alimentos para os trabalhadores representou, positivamente, uma frequência aumentada do consumo desses alimentos (Himberg-Sundet et al., 2019). Dessa forma, a disponibilidade de alimentos in natura pode representar uma estratégia de promoção da saúde no âmbito do trabalho.

\subsection{Implicações para futuras pesquisas e para uma política de promoção da saúde do trabalhador}

Atualmente, não há uma política específica em promoção da saúde do trabalhador e da trabalhadora, no Brasil. Há no país, uma Política Nacional de Saúde do Trabalhador e da Trabalhadora (Brasil, 2012) e uma Política Nacional de Promoção da Saúde (Brasil, 2014) para a população, de uma forma ampla, e não especificamente para o grupo de trabalhadores. Diante dos resultados trazidos nesta revisão, foi construído o quadro comparativo (Quadro 3) sobre quais aspectos não estão englobados nessas políticas e que poderiam fazer parte, numa futura reformulação.

Quadro 3: Comparativo entre os resultados encontrados nessa revisão e como são discutidos nas políticas atuais, no Brasil.

\begin{tabular}{|c|c|c|}
\hline $\begin{array}{l}\text { Principais resultados da } \\
\text { revisão }\end{array}$ & $\begin{array}{c}\text { Política Nacional de Saúde do Trabalhador e da } \\
\text { Trabalhadora } \\
\end{array}$ & Política Nacional de Promoção da Saúde \\
\hline $\begin{array}{l}\text { Sentidos do trabalho e } \\
\text { autonomia do(a) } \\
\text { trabalhador(a) }\end{array}$ & $\begin{array}{l}\text {-Não explora o sentido do trabalho; } \\
\text {-Não traz nenhum aspecto da relação autonomia, } \\
\text { trabalho e saúde. }\end{array}$ & $\begin{array}{l}\text {-Não traz nenhum aspecto do sentido do trabalho; } \\
\text {-Em relação à autonomia, não há descrição específica sobre } \\
\text { o trabalho, mas essa política define como um dos objetivos } \\
\text { específicos o desenvolvimento de habilidades pessoais e } \\
\text { competências para a promoção da saúde para a autonomia } \\
\text { dos sujeitos. }\end{array}$ \\
\hline $\begin{array}{l}\text { Território e acesso aos } \\
\text { serviços de saúde }\end{array}$ & $\begin{array}{l}\text {-Descreve como pressupostos do fortalecimento da } \\
\text { Vigilância em Saúde do Trabalhador e demais } \\
\text { componentes da Vigilância em Saúde, a identificação } \\
\text { das atividades produtivas no território e das demandas e } \\
\text { necessidades de saúde dos trabalhadores no território; } \\
\text {-Descreve como estratégia o mapeamento das atividades } \\
\text { produtivas e seu impacto ambiental no território; } \\
\text {-Descreve também como estratégia a investigação de } \\
\text { surtos ou outros eventos decorrentes dos impactos } \\
\text { ambientais das atividades produtivas no território; } \\
\text {-E a identificação das atividades produtivas e o perfil da } \\
\text { população trabalhadora no território. }\end{array}$ & $\begin{array}{l}\text {-Entende como tema transversal da política os ambientes e } \\
\text { territórios saudáveis, neles incluídos os do trabalho, } \\
\text { devendo identificar oportunidades para ações de promoção } \\
\text { da saúde de forma participativa e dialógica; } \\
\text {-Descreve como necessidade a articulação entre todos os } \\
\text { equipamentos de produção de saúde do território; } \\
\text {-A política de promoção da saúde deve se adequar as } \\
\text { especificidades locorregionais (e não o contrário) e } \\
\text { considerar as necessidades do seu território; } \\
\text {-O acesso é tratado de forma ampla, como valor fundante da } \\
\text { política para que esteja ao alcance de todos os benefícios da } \\
\text { vida em sociedade. }\end{array}$ \\
\hline $\begin{array}{l}\text { Relações interpessoais no } \\
\text { trabalho }\end{array}$ & $\begin{array}{l}\text {-Não descreve nenhum aspecto explicito das relações } \\
\text { interpessoais no trabalho. Traz apenas uma questão mais } \\
\text { geral, como ação de Saúde do Trabalhador na Atenção } \\
\text { Primária que é a identificação da rede de apoio social } \\
\text { aos trabalhadores no território. }\end{array}$ & $\begin{array}{l}\text {-Não descreve especificamente sobre o trabalho, mas define } \\
\text { as relações construídas entre os sujeitos e coletivos como } \\
\text { um elemento para a felicidade. }\end{array}$ \\
\hline Gênero e trabalho & $\begin{array}{l}\text {-Não traz nenhum aspecto da questão de gênero, } \\
\text { especificamente. Apenas sinaliza que a política inclui } \\
\text { todos os sujeitos, sem distinções. }\end{array}$ & $\begin{array}{l}\text {-Descreve como valores fundantes no processo de } \\
\text { efetivação da política: "o respeito às diversidades }[\ldots] \text { de } \\
\text { gênero [...]"; } \\
\text {-Descreve também como objetivos específicos: “[...] a } \\
\text { adoção de práticas sociais e de saúde }[\ldots] \text { visando reduzir as } \\
\text { desigualdades }[\ldots . .] \text { de gênero }[\ldots . .] \text { "; } \\
\text {-E descreve como um dos temas prioritários "a promoção da } \\
\text { cultura de paz e de direitos humanos }[. . .] \text { que estimulem }[\ldots] \\
\text { o respeito às diversidades e diferenças de gênero." }\end{array}$ \\
\hline $\begin{array}{l}\text { Condições de trabalho: } \\
\text { jornada e esforço físico }\end{array}$ & $\begin{array}{l}\text {-A única ação englobada dentro das condições de } \\
\text { trabalho diz respeito ao fortalecimento das articulações } \\
\text { intersetoriais, por meio da fiscalização conjunta onde } \\
\text { houver situações insalubres, perigosas ou degradantes, } \\
\text { pelos Ministérios. }\end{array}$ & $\begin{array}{l}\text {-Não descreve nenhum aspecto das condições de trabalho. } \\
\text { Apenas, traz que a política objetiva promover a melhoria } \\
\text { das condições e modos de viver. }\end{array}$ \\
\hline $\begin{array}{l}\text { Condições de trabalho: } \\
\text { acesso à alimentação } \\
\text { saudável no local de trabalho }\end{array}$ & $\begin{array}{l}\text {-Não traz nenhum aspecto sobre o acesso à alimentação } \\
\text { saudável no trabalho. }\end{array}$ & $\begin{array}{l}\text { - Descreve, de uma forma geral, como tema prioritário da } \\
\text { política a garantia do direito humano à alimentação } \\
\text { adequada. }\end{array}$ \\
\hline
\end{tabular}

Fonte: Autores. 
Em síntese, destaca-se como temas para uma futura política, ou programa de promoção da saúde do trabalhador e da trabalhadora a questão gênero no trabalho, as condições de trabalho (e o acesso à alimentação saudável), as relações interpessoais no trabalho e os sentidos do trabalho. De acordo com a revisão realizada e o quadro comparativo apresentado das políticas mais próximas do tema discutido nesta pesquisa, essas são as questões relevantes para suprir as lacunas existentes.

\section{Considerações Finais}

Por meio desta revisão foi possível mapear os principais resultados de estudos qualitativos sobre promoção da saúde do trabalhador e as lacunas existentes nas políticas nesse campo de estudo, no Brasil. Sabendo-se que as pesquisas qualitativas proporcionam análises em profundidade, este estudo proporcionou trazer para discussão temas diversos, ideal para uma política que precisa ir ao encontro de problemas, provavelmente, não discutidos por métodos quantitativos. As relações interpessoais no trabalho, a questão de gênero, os sentidos do trabalho e as condições de trabalho ganharam destaque ao se comparar com os dispositivos legais vigentes, pela ausência de previsão dessas questões.

Para futuras pesquisas, sugere-se que seja realizado um comparativo com estudos internacionais, apontando avanços e oportunidades de melhoria, se comparados com o cenário brasileiro, no campo da promoção da saúde do trabalhador. Com estes dados será possível ampliar o conhecimento na área e trazer elementos importantes para a implementação de uma política pública brasileira em prol da saúde do trabalhador e da trabalhadora.

\section{Referências}

Alcantara, V. C. G. de, Silva, R. M. C. R. A., Pereira, E. R., Silva, D. M. da, \& Flores, I. P. (2020). O trânsito na compreensão de motoristas de ônibus: Possibilidades do cuidado interdisciplinar. Research, Society and Development, 9(3), e36932369-e36932369. https://doi.org/10.33448/rsd-v9i3.2369

Amaral, M. M. S., Escóssia, L. da, Amaral, M. M. S., \& Escóssia, L. da. (2021). Por uma clínica de(s) território no contexto do SUS. Fractal: Revista de Psicologia, 33(1), 31-40. https://doi.org/10.22409/1984-0292/v33i1/5782

Araújo, T. M. de, Godinho, T. M., Reis, E. J. F. B. dos, \& Almeida, M. M. G. de. (2006). Diferenciais de gênero no trabalho docente e repercussões sobre a saúde. Ciência \& Saúde Coletiva, 11, 1117-1129. https://doi.org/10.1590/S1413-81232006000400032

Araújo, T. M. de, Pinho, P. de S., Masson, M. L. V., Araújo, T. M. de, Pinho, P. de S., \& Masson, M. L. V. (2019). Trabalho e saúde de professoras e professores no Brasil: Reflexões sobre trajetórias das investigações, avanços e desafios. Cadernos de Saúde Pública, 35. https://doi.org/10.1590/0102$311 \times 00087318$

Arksey, H., \& O’Malley, L. (2005). Scoping studies: Towards a methodological framework. International Journal of Social Research Methodology, 8(1), 1932. https://doi.org/10.1080/1364557032000119616

Bezerra, M. das G. V., Rigotto, R. M., Pessoa, V. M., \& Silva, F. V. E. da. (2014). Implicações do desenvolvimento econômico no trabalho, ambiente e saúde em comunidades portuárias no Ceará, Brasil. Ciência \& Saúde Coletiva, 19(10), 4023-4030. https://doi.org/10.1590/1413-812320141910.09802014

Brasil. (1988). Constituição Federal de 1988. Presidência da República. http://www.planalto.gov.br/ccivil_03/constituicao/constituicao.htm

Brasil. (2002). As Cartas de Promoção da Saúde. Ministério da Saúde, Secretaria de Políticas de Saúde, Projeto Promoção da Saúde. - Brasília: Ministério da Saúde. https://bvsms.saude.gov.br/bvs/publicacoes/cartas_promocao.pdf

Brasil. (2012). Portaria $n^{o}$ 1.823, de 23 de AGOSTO de 2012. Institui a Política Nacional de Saúde do Trabalhador e da Trabalhadora. https://bvsms.saude.gov.br/bvs/saudelegis/gm/2012/prt1823_23_08_2012.html

Brasil. (2014). Portaria $n^{o}$ 2.446, de 11 de novembro de 2014. Redefine a Política Nacional de Promoção da Saúde (PNPS). http://bvsms.saude.gov.br/bvs/saudelegis/gm/2014/prt2446_11_11_2014.html

Buss, P. M., \& Carvalho, A. I. de. (2009). Desenvolvimento da promoção da saúde no Brasil nos últimos vinte anos (1988-2008). Ciência \& Saúde Coletiva, 14, 2305-2316. https://doi.org/10.1590/S1413-81232009000600039

Camara, E. A. R. da, Belo, M. S. da S. P., Peres, F., Camara, E. A. R. da, Belo, M. S. da S. P., \& Peres, F. (2020). Desafios e oportunidades para a formação em Saúde do Trabalhador na Atenção Básica à Saúde: Subsídios para estratégias de intervenção. Revista Brasileira de Saúde Ocupacional, 45. https://doi.org/10.1590/2317-6369000009418

Cardoso, A. C., \& Morgado, L. (2019). Trabalho e saúde do trabalhador no contexto atual: Ensinamentos da Enquete Europeia sobre Condições de Trabalho. Saúde e Sociedade, 28, 169-181. https://doi.org/10.1590/s0104-12902019170507 
Cocchiara, R. A., Dorelli, B., Gholamalishahi, S., Longo, W., Musumeci, E., Mannocci, A., \& La Torre, G. (2020). Tai Chi and Workplace Wellness for Health Care Workers: A Systematic Review. International Journal of Environmental Research and Public Health, 17(1). https://doi.org/10.3390/ijerph17010343

Coelho, A. P. F., Beck, C. L. C., Fernandes, M. N. da S., Prestes, F. C., \& Silva, R. M. da. (2016). Risco de adoecimento relacionado ao trabalho e estratégias defensivas de mulheres catadoras de materiais recicláveis. Escola Anna Nery, 20(3). https://doi.org/10.5935/1414-8145.20160075

Creswell, J. W. (2014). Investigação Qualitativa e Projeto de Pesquisa: Escolhendo entre Cinco Abordagens (3a ed.). Penso.

Dooris, M. (2006). Healthy settings: Challenges to generating evidence of effectiveness. Health Promotion International, 21(1), 55-65. https://doi.org/10.1093/heapro/dai030

Feliciano, K. V. de O., Kovacs, M. H., \& Sarinho, S. W. (2011). Burnout entre médicos da Saúde da Família: Os desafios da transformação do trabalho. Ciência \&amp; Saúde Coletiva, 16(8), 3373-3382. https://doi.org/10.1590/S1413-81232011000900004

Ferreira, E. S., Souza, M. B. de, Souza, N. V. D. de O., Tavares, K. F. A., \& Pires, A. da S. (2015). A relevância do cuidado de si para profissionais de enfermagem. Ciênc. Cuid. Saúde, 14(1), 978-992.

Gomes, M. F., Lima, A. da S. R. de, Feitosa, L. dos S., Pontes Netto, V. B., Nascimento, R. D. do, \& Andrade, M. S. (2015). Riscos e agravos ocupacionais: Percepções dos agentes comunitários de saúde. Rev. Pesqui. Cuid. Fundam. (Online), 7(4), 3574-3586.

Grimani, A., Aboagye, E., \& Kwak, L. (2019). The effectiveness of workplace nutrition and physical activity interventions in improving productivity, work performance and workability: A systematic review. BMC Public Health, 19(1), 1676. https://doi.org/10.1186/s12889-019-8033-1

Hawker, S., Payne, S., Kerr, C., Hardey, M., \& Powell, J. (2002). Appraising the Evidence: Reviewing Disparate Data Systematically. Qualitative Health Research, 12(9), 1284-1299. https://doi.org/10.1177/1049732302238251

Himberg-Sundet, A., Kristiansen, A. L., Gebremariam, M. K., Moser, T., Andersen, L. F., Bjelland, M., \& Lien, N. (2019). Exploring the workplace climate and culture in relation to food environment-related factors in Norwegian kindergartens: The BRA-study. PLOS ONE, 14(12), e0225831. https://doi.org/10.1371/journal.pone.0225831

Hirata, H., \& Kergoat, D. (2008). Divisão sexual do trabalho profissional e doméstico: Brasil, França, Japão. Mercado de trabalho e gênero: comparações internacionais. Rio de Janeiro: FGV, 263-278.

Jaskowiak, C. R., \& Fontana, R. T. (2015). O trabalho no cárcere: Reflexões acerca da saúde do agente penitenciário. Revista Brasileira de Enfermagem, 68(2), 235-243. https://doi.org/10.1590/0034-7167.2015680208i

Lacaz, F. A. de C. (2000). Qualidade de vida no trabalho e saúde/doença. Ciência \& Saúde Coletiva, 5, 151-161. https://doi.org/10.1590/S141381232000000100013

Lake, A. A., Smith, S. A., Bryant, C. E., Alinia, S., Brandt, K., Seal, C. J., \& Tetens, I. (2016). Exploring the dynamics of a free fruit at work intervention. BMC Public Health, 16. https://doi.org/10.1186/s12889-016-3500-4

Leônidas, S. R., \& Melo, C. de F. (2018). Avaliação do Centro de Referência em Saúde do Trabalhador Cearense pelos Usuários e Representante dos Sindicatos. Psico-USF, 23(1), 127-138. https://doi.org/10.1590/1413-82712018230111

Macedo, A. P., Cruz, J. M., \& Ribeiro, J. M. (2018). Promover a saúde no novo paradigma. Revista Brasileira em Promoção da Saúde, 31(0), Article 0. https://doi.org/10.5020/18061230.2018.8649

Michelin, S. R., Nitschke, R. G., Tholl, A. D., Laureano, D. D., Silva, K. M., \& Potrich, T. (2016). O quotidiano dos trabalhadores da atenção básica: Limites para a promoção da saúde. Ciênc. cuid. saúde, 15(4). https://doi.org/10.4025/cienccuidsaude.v15i4.32600

Morin, E. (2002). Os sentidos do trabalho. In Wood Jr T. Gestão empresarial: O fator humano. (p. 13-34). Atlas.

Motalebi G., M., Keshavarz Mohammadi, N., Kuhn, K., Ramezankhani, A., \& Azari, M. R. (2018). How far are we from full implementation of health promoting workplace concepts? A review of implementation tools and frameworks in workplace interventions. Health Promotion International, 33(3), 488504. https://doi.org/10.1093/heapro/daw098

Munn, Z., Peters, M. D. J., Stern, C., Tufanaru, C., McArthur, A., \& Aromataris, E. (2018). Systematic review or scoping review? Guidance for authors when choosing between a systematic or scoping review approach. BMC Medical Research Methodology, 18(1), 143. https://doi.org/10.1186/s12874-018-0611-X

Organização Internacional do Trabalho. (2018). Condições de Trabalho. http://www.ilo.org/lisbon/temas/WCMS_650796/lang--pt/index.htm

Patel, R., \& Moonesinghe, S. R. (2020). A seat at the table is no longer enough: Practical implementable changes to address gender imbalance in the anaesthesia workplace. British Journal of Anaesthesia, 124(3), e49-e52. https://doi.org/10.1016/j.bja.2019.12.029

Pennisi, P. R. C., Alves, N. C., Michelin, P. S., Medeiros-Souza, L., Herval, Á. M., Paranhos, L. R., Pennisi, P. R. C., Alves, N. C., Michelin, P. S., MedeirosSouza, L., Herval, Á. M., \& Paranhos, L. R. (2020). Qualidade de vida de profissionais da Saúde da Família: Uma revisão sistemática e metassíntese. Revista Brasileira de Enfermagem, 73(5). https://doi.org/10.1590/0034-7167-2019-0645 
Research, Society and Development, v. 11, n. 1, e19811125140, 2022

(CC BY 4.0) | ISSN 2525-3409 | DOI: http://dx.doi.org/10.33448/rsd-v11i1.25140

Poland, B. (2007). Health Promotion in Canada: Perspectives \& Future Prospects. Revista Brasileira em Promoção da Saúde, $20(1), 3-11$.

Proper, K. I., \& van Oostrom, S. H. (2019). The effectiveness of workplace health promotion interventions on physical and mental health outcomes - a systematic review of reviews. Scandinavian Journal of Work, Environment \& Health, 45(6), 546-559. https://doi.org/10.5271/sjweh.3833

Ribeiro, J., Souza, D. N. de, \& Costa, A. P. (2016). Investigação qualitativa na área da saúde: Por quê? Ciência \& Saúde Coletiva, 21, 2324-2324. https://doi.org/10.1590/1413-81232015218.15612016

Santos, F. B. dos, Lourenção, L. G., Vieira, E., Ximenes Neto, F. R. G., Oliveira, A. M. N. de, Oliveira, J. F. de, Borges, M. A., \& Arroyo, T. R. (2021). Estresse ocupacional e engajamento no trabalho entre policiais militares. Ciência \& Saúde Coletiva, 26, 5987-5996. https://doi.org/10.1590/1413812320212612.14782021

Santos, J. C. B. dos, \& Hennington, É. A. (2013). Aqui ninguém domina ninguém: Sentidos do trabalho e produção de saúde para trabalhadores de assentamento do Movimento dos Trabalhadores Rurais Sem Terra. Cadernos de Saúde Pública, 29(8), 1595-1604. https://doi.org/10.1590/0102$311 \mathrm{X} 00096612$

Santos, M. (2005). O retorno do território. In: OSAL: Observatorio Social de América Latina. Buenos Aires: CLACSO. p. 251-261. http://biblioteca.clacso.edu.ar/ar/libros/osal/osal16/32Santo.pdf

Scott, J. (1990). Gênero: Uma categoria útil na Pesquisa Histórica. Educação e Realidade. Porto Alegre, 16(2).

Silva, D. P. da, Freitas, R. F., Souza, L. F. de, Teixeira, N. A., Dias, E. C., \& Rocha, J. S. B. (2021). Práticas profissionais em saúde do trabalhador na Atenção Primária: Desafios para implementação de políticas públicas. Ciência \& Saúde Coletiva, 26, 6005-6016. https://doi.org/10.1590/1413812320212612.14842021

Silva, S. R. A. da, Amorim, R. da C., \& Almeida, A. M. de. (2015). Percepção de feirantes hipertensos sobre o adoecer crônico [Hypertensive street market vendors' perceptions of chronic illness]. Revista Enfermagem UERJ, 23(6), 761-766. https://doi.org/10.12957/reuerj.2015.8494

Soares, J. E. M., Santana, J. F. C. L. de, Vasconcelos, R. M. A., Santos, P. S., Rocha, M. V. S., \& Apolinário, T. A. (2021). Saúde do trabalhador: Um olhar para o Centro Referencial de Saúde. Research, Society and Development, 10(3), e50710313589-e50710313589. https://doi.org/10.33448/rsd-v10i3.13589

Tricco, A. C., Lillie, E., Zarin, W., O’Brien, K. K., Colquhoun, H., Levac, D., Moher, D., Peters, M. D. J., Horsley, T., Weeks, L., Hempel, S., Akl, E. A., Chang, C., McGowan, J., Stewart, L., Hartling, L., Aldcroft, A., Wilson, M. G., Garritty, C., \& Straus, S. E. (2018). PRISMA Extension for Scoping Reviews (PRISMA-ScR): Checklist and Explanation. Annals of Internal Medicine, 169(7), 467-473. https://doi.org/10.7326/M18-0850

Viana, E. A. de S., \& Machado, M. N. da M. (2011). Sentido do trabalho no discurso dos trabalhadores de uma ONG em Belo Horizonte. Psicologia \&amp; Sociedade, 23(1), 46-55. https://doi.org/10.1590/S0102-71822011000100006

World Health Organization. (2008). Preventing Noncommunicable Diseases in the Workplace through Diet and Physical Activity. Global Strategy on Diet, Physical Activity and Health. https://www.who.int/dietphysicalactivity/workplace/en/

Ziliotto, D. M., \& Jaques, A. G. (2017). As contingências do trabalho bancário: Um estudo sobre os mecanismos de mediação e defesa do sofrimento. Interação em Psicologia, 21(1), Article 1. https://doi.org/10.5380/psi.v21i1.45308 